\title{
Data-driven method for mobile game publishing revenue forecast
}

\author{
Yanhui Su$^{1}\left(\mathbb{D} \cdot\right.$ Per Backlund $^{1} \cdot$ Henrik Engström $^{1}$ \\ Received: 16 June 2021 / Revised: 28 November 2021 / Accepted: 4 December 2021 / Published online: 23 December 2021 \\ (c) The Author(s) 2021
}

\begin{abstract}
Games as a service is similar to software as a service, which provides players with game content on a continuous monetization model. Game revenue forecast is vital to game developers to make the right business decisions, such as determining the marketing budget, controlling the development cost, and setting up benchmarks for evaluating game publishing performance. How to make the revenue forecast and integrate it with the game publishing process is hard for small and medium-sized independent (indie) game developers. This includes all steps of the process, from forecasting to decision-making based on the results. This paper provides a data-driven method that uses the mobile game revenue forecast based on different time-series prediction models to drive the game publishing. We demonstrate how to use the data-driven method to guide an indie game studio to forecast revenue and then set the revenue forecast as the internal benchmark to drive game publishing. In practice, we involve a real game project from an indie game studio and provide guidance for one of their casual game projects. Then, based on the revenue forecast, we discuss how to set the revenue forecast as an internal benchmark and drive the actions for mobile game publishing. Finally, we make a conclusion on how our data-driven method can be used to drive mobile game publishing and also discuss future research work.
\end{abstract}

Keywords Business Intelligence · Game analytics · Game metrics · Indie game developer · Benchmark · Time-series prediction $\cdot$ Game publishing

\section{Introduction}

In the current state of the industry, game is considered as a service and not as a finished product anymore, which through regular updates and the use of data, and then becomes good and meets a certain amount of success [1]. According to the survey report [2], the mobile game revenues account for $49 \%$ of the global game market, which had reached $\$ 86.3$ billion during the past year. In alignment, as more and more mobile games appear, we also see an increasing number of small and medium-sized independent (indie) game developers who have limited resources to do game development. However, most indie game developers are good at game development, but they lack experience concerning game publishing [3]. Game publishing can be recognized as an important channel

Yanhui $\mathrm{Su}$

yanhui.su@his.se

Per Backlund

per.backlund@his.se

Henrik Engström

henrik.engstrom@his.se

1 School of Informatics, University of Skövde, Skövde, Sweden for connecting the game and the target players. Traditionally, publishing has been handled by separate actors on the market [4]. In the past, the game business was dominated by big companies, such as Nintendo and Sony. It was hard for indie game developers to compete with these giants as they controlled the whole game industry value chain from hardware development to the game publishing channels. However, with the appearance of mobile game devices and third-party game publishing channels, such as App Store and Google Play, indie game developers can launch games on all kinds of app stores themselves. These changes bring a new phenomenon to the game business and the game industry has moved toward a new situation where indie mobile game developers start to learn and do game publishing themselves.

Analytics is a subset of Business Intelligence (BI) that uses data to understand and analyze business performance. It is meant to ensure that the money and resources devoted to marketing focus on the most effective campaigns and channels [5]. Game analytics has already been used in the game industry for many years. Kim et al. [6] discuss how game analytics can be used to identify in-game balancing issues. Hullett et al. [7] use it to reduce game development costs and avoid risks 
in game development. Moura et al. [8] apply it to visualize players' movement paths on the map and identify the blocking points from the player side. Zoeller [9] also provides a solution to detect in-game bugs by game analytics. However, game analytics related to mobile game research, from the academic side, is fragmented. As most of such research focuses on game development, the focus on game publishing is scarce. Considering mobile game analytics, Drachen et al. [10] point out this field of research is in its infancy and the available knowledge is heavily fragmented.

During mobile game publishing, game developers face issues on how to do a revenue forecast for their games [11]. Based on the revenue forecast, they can make a business plan for marketing promotion, such as how much marketing budget needs to be used for new user acquisition channels based on the ROI (Return on Investment). It is also possible to follow up on game development costs and set up benchmarks for evaluating game publishing performance. However, making and utilizing the revenue forecast is hard, especially for indie game developers who have little or no revenue forecast experience. Based on our previous interviews with indie game studios, most of them have issues with related game data collection, data analysis and prediction during their game publishing process [12]. As for the term data-driven, it describes a business state where data are used to power decision-making and related activities efficiently. Data-driven characteristics include well-integrated metrics and related algorithms [13]. As for the benchmark, it is used to identify the practices that adopted and implemented by the organization to improve company performance [14]. The internal benchmarks, which can be shared internally because of their sensitive business data, can be used for specific operations [15].

In this paper, we suggest a data-driven method to guide indie game developers to make revenue forecast and set it as an internal benchmark and drive their game publishing process. We use the forecasted revenue as a benchmark to judge the actual effect of game publishing, and when there is a gap between the forecast and the real revenue, suggestions are provided for indie game developers to take action to optimize. In practice, we target F2P (Free-to-play) game, the mainstream mobile game model, and provide a method for indie mobile game developers to predict their launched game's revenue first and then set the revenue forecast as an internal benchmark to drive their game publishing and guide actions. In Sect. 2, we discuss related work with game analytics and revenue predictions. Section 3 provides the methodology for our research, which focuses on a case study of an indie game revenue forecast and describes the research process, including four steps. In Sect. 4, we apply the method for mobile game revenue forecast by means of six actions to set an internal benchmark and compare it with the real revenue to evaluate the game publishing performance. In Sect. 5,

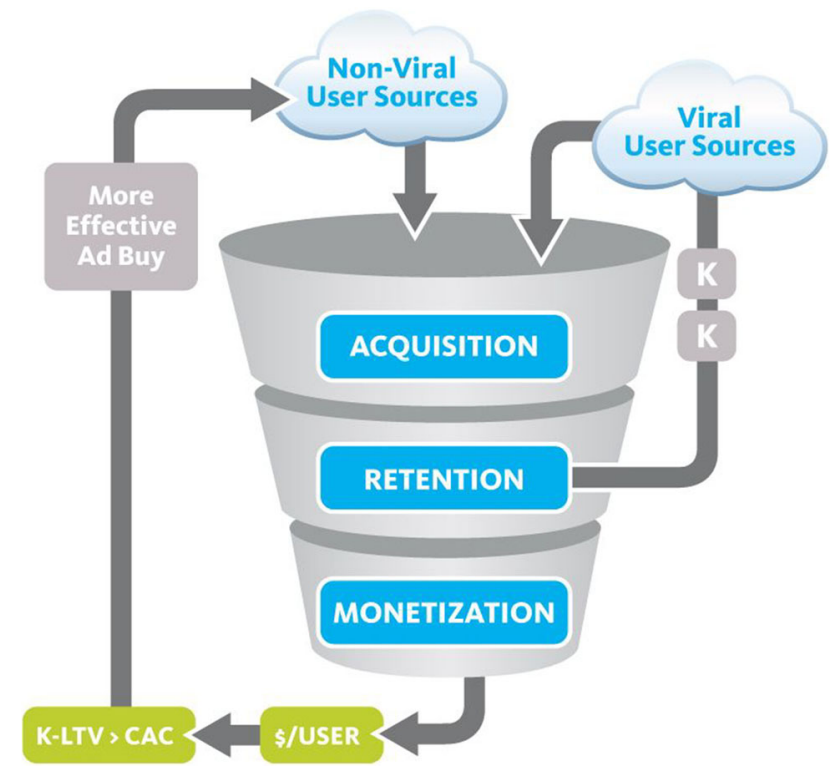

Fig. 1 ARM funnel model [18]

we provide a detailed analysis of the reasons if the revenue cannot meet the benchmark and propose actions for improvement. In Sect. 6, we conclude how our data-driven method can be used for the revenue forecast and drive the game publishing business and discuss future research work.

\section{Related work}

At present, based on the literature review, most of the game analytics mainly focus on game development and game research [16]. However, the application of game analytics in mobile game publishing is highly fragmented [10]. Moreira et al. [17] use the ARM (acquisition, retention, and monetization) funnel model as the basic analysis for the game publishing process. However, this funnel model is originally developed for social games by the company Kontagent [18]. It can be used for visualizing the mobile game publishing process by the three stages: acquisition, retention, and monetization, as shown in Fig. 1.

However, the ARM funnel model mainly focuses on the viral $\mathrm{K}$ factor promotion effect if K-LTV (Life Time Value) is great than CAC (Customer Acquisition Cost). It only evaluates how the game performance changes by tracking these three stages. The potential issues beyond acquisition, retention, and monetization cannot be solved by the ARM funnel model. For example, it is hard to see what is related to game revenue from this model, especially where the revenue comes from and the relationship between the revenue and also the player behaviors. It does not provide revenue forecast and benchmark tracking solutions as well. 
Our research goal in this paper is to extend the ARM funnel model for mobile game publishing and provide a data-driven method for mobile games. This method can forecast revenue for indie game developers and set it as the internal benchmark to drive their mobile game publishing. For game revenue forecast, most predictions are based on the traditional game revenue forecast method [19], which depends on the game revenue formula, as shown in below formula 1 :

$$
\text { Revenue }=A R P U \times M A U
$$

MAU represents the number of Monthly Active Users, and ARPU stands for Average Revenue Per User. Generally, the MAU and ARPU will decrease to a certain extent as it is normal that game performance falls year by year with the game lifetime. Therefore, when the game developers rely on this traditional method to do the revenue forecast, they usually estimate a certain degree of decline in ARPU and MAU. However, this estimation method relies too much on personal experience, especially concerning how much ARPU and MAU percentage should be decreased to get the proper revenue forecast compared with previous revenue. It is hard to get a reliable revenue forecast. As for indie game developers, based on our previous interviews, they have challenges related to game data collection, data analysis, and prediction during game publishing [12]. It is difficult for them to clearly understand the meaning behind the data and improve the publishing performance. So compared with the traditional game revenue forecast method, which needs more publishing experiences, how to provide a reliable revenue forecast for the indie game developers and drive mobile game publishing is the big challenge.

As for the related prediction research, most focus on churn prediction and player purchase behavior prediction. Runge et al. [20] point out that predicting when a player will leave a game creates a unique opportunity to increase the player's lifetime and revenue. Periáñez et al. [21] develop a survival integration model for social games that provides comprehensive churn prediction. As for the related revenue prediction research, Sifa et al. [22] focus on predicting future purchase activities by formulating the process as the player classification with a regression problem and providing the solution for purchase behavior prediction. Xie et al. [23] focus on predicting the first purchase behaviors in two social games. They start to use the frequency of game events as data representations to predict the first purchase. However, the previous game predictions primarily focused on predicting player churn and purchase behaviors, which lacks the direct forecast about game revenue, especially based on the historical revenue data. Besides this, Drachen et al. [10, p.3] also point out, "A general observation across these studies is that they introduce individual problem definitions, and report data and methods differently, indicating a lack of standards

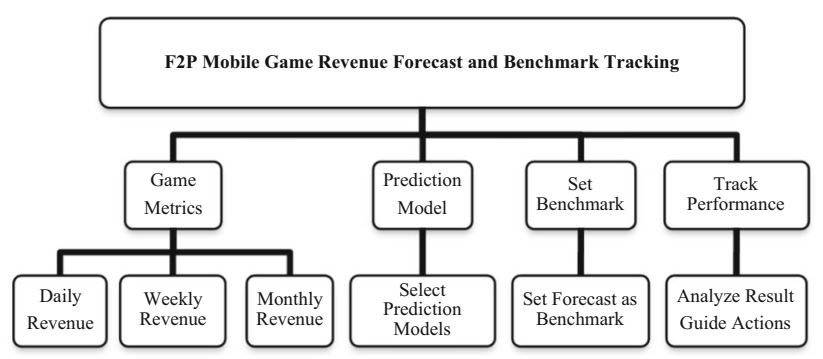

Fig. 2 F2P mobile game revenue forecast and benchmark tracking

for reporting work in mobile game analytics." That is why we plan to provide a data-driven method that involves the time-series prediction models for mobile game publishing revenue forecast. This revenue forecast is based on historical revenue data and uses the time-series prediction models [24]. It is reliable compared with the traditional method based on personal experience [19].

As data-driven describes a business state where data are used for decision-making and related activities, it includes well-integrated metrics and related algorithms for analysis and guides actions. We design and provide the method for the F2P mobile game revenue forecast and benchmark tracking. It includes the game metrics and prediction models, setting the internal benchmark, tracking the performance, and guiding actions, as shown in Fig. 2. We involve Daily Revenue (DR), Weekly Revenue (WR), and also Monthly Revenue (MR) metrics for the prediction. All three of these metrics can be used to make predictions. Choosing DR, WR, or MR to make a revenue forecast depends on whether the goal is to get a daily, weekly, or monthly forecast.

As for the prediction models, Markovska et al. [25] made a comparative study of ARIMA (Autoregressive Integrated Moving Average) and Holt-Winters when to use for statistical prediction. They point out that the ARIMA gives more accurate results when the forecast is based on daily time-series data, and the Holt-Winters has better performance for weekly and monthly time series data. Holt-Winters is a widely used time-series prediction model. The value of the Holt-Winters prediction reflects the long-term trend of the indicator and shows its seasonal changes [26]. In practice, many mobile game revenues are subject to seasonal influences. As an example, usually, the revenue increases during holidays such as Christmas. That is also the reason why Holt-Winters seasonal model is appropriate for game revenue prediction. In order to verify the prediction result, we plan to use both the Holt-Winters and ARIMA models for the revenue forecast first. Then, we can compare each prediction result with the real revenue and make a conclusion.

In addition to comparing the revenue forecast with the real revenue, the most valuable part is that we set the revenue forecast as an internal benchmark for indie developers to 
evaluate whether their game publishing revenue target meets this benchmark. If not, we provide the solutions to analyze the reasons and take action to improve the game performance. The reason why we want to use the revenue prediction as an internal benchmark to drive the indie game publishing is that comparing results with benchmark makes it easier to detect potential gaps and also gives valuable advice and a deeper understanding when it comes to which parts need to be improved and how to implement [27].

\section{Research method and process}

A case study is an empirical investigation of an individual case used to investigate a problem and discover the reasons behind the phenomenon. Cousin [28] claims that case study research investigates and interprets a scenario intending to increase the apprehension. Usually, a case study can be recognized as a qualitative research method [29]. This research aims to solve indie game developers' revenue forecast issues, provide a data-driven method with related metrics and prediction models for indie mobile game revenue forecast, and then set the reliable forecast as an internal benchmark to drive their mobile game publishing.

We cooperate with an indie game studio with less than ten employees for this case study and participate in a specific mobile games publishing project. The first author worked with the indie game studio, applied the method to their mobile game project, and guided them to use the predicted results as an internal benchmark to drive their game publishing. From the research ethics side, we got approval from the indie game studio to use related data and materials for our research as long as we provide free guidance to their game project.

For our research process, we first propose the method to predict mobile game revenue combined with related metrics and the time-series prediction models. Second, based on the revenue forecast, we set the reliable forecast as an internal benchmark and drive the game publishing. We introduce and apply the data-driven method for using metrics and timeseries prediction models to predict the revenue of a casual mobile game released by the indie game studio and then set the revenue forecast as an internal benchmark. This benchmark can be used to identify the game publishing potential problems and improve the game performance. Based on the internal benchmark, we also guide the indie game developer to analyze and evaluate whether the real revenue can meet the benchmark or not and determine the reasons behind that. The whole research process consists of four steps.

- Step 1 Game developer selection According to the research goal, we first propose the new method for indie mobile game revenue forecast only based on the historical revenue. We found an indie game studio based in a Swedish
Game Incubator that had published mobile games themselves. The indie game studio has already launched a casual game for more than three years but has no revenue forecast experience. So, they form a suitable case to do the revenue forecast.

- Step 2 Introduce the method To ensure that the indie game developer is familiar with our data-driven method, the first author visited the studio and introduced the method, especially how it can be used to forecast revenue and drive game publishing. In practice, the first author held a meeting with the indie game studio, explained the method to them, and introduced the related metrics and the prediction models for the revenue forecast.

- Step 3 Apply the method In order to solve the specific issues about revenue forecast faced by indie game developers, we guide the indie game studio to use the method in their mobile game publishing to make the revenue forecast for their launched game and then set the forecast as the internal benchmark to drive their game publishing. In practice, the first author worked and helped them collect the related revenue data and make the prediction. The metrics define what kind of data needs to be collected for the revenue forecast. The model focuses on how to analyze data and make a prediction.

- Step 4 Analysis and suggestions Based on the revenue forecast, we compare it with real revenue and guide the indie game developer to analyze and evaluate whether the real revenue can hit the benchmark or not and determine the reasons behind that. In practice, we explore how to set our predicted result as an internal benchmark to guide the indie game studio for mobile game publishing, such as the monthly mobile game publishing performance evaluation, game version optimization suggestions, and marketing promotion budget control.

\section{Apply the data-driven method}

In order to make it feasible for the indie game developer to use our data-driven method to make revenue forecast and set the forecast as the internal benchmark to drive their mobile game publishing process, we design a revenue forecast and benchmark tracking application actions. As shown in Fig. 3, the data-driven method application includes six actions presented in the following sub-sections.

\subsection{Select metrics}

The first action when using our method for game revenue forecast is to select metrics based on the forecast goal. Determining the goals of the forecast is critical to the final results. As for the indie game studio, the forecasting goal is to predict the following year's revenue performance through existing 


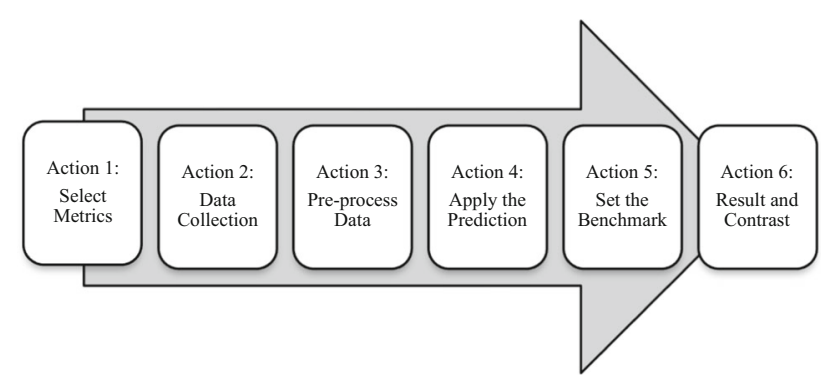

Fig. 3 The data-driven method application

historical data. Based on the guideline, we need to decide whether to choose DR, WR, or MR to make a revenue forecast. This decision depends on whether the goal is to get a daily, weekly, or monthly forecast. The indie game studio was eager to get a monthly revenue forecast as a revenue benchmark for evaluating monthly game performance. We hence chose the MR metrics to make a revenue forecast.

\subsection{Data collection}

The second action when using our method for game revenue forecast is to collect data based on the forecast goal. The first author worked with the indie game studio to obtain their game revenue data. They want to predict the monthly revenue for their existing game. So they only provide monthly revenue data about their specific existing casual game over the past two years, from 2017 to 2018 . Based on these monthly data, we predict the revenue for the following year. To further compare and set our prediction as an internal benchmark, we collaborated with the indie game studio again to obtain real revenue data for the following year and guide the indie mobile game publishing by comparing them with revenue prediction based on the data-driven method.

After getting the two years of historical game revenue, we can plot the entire revenue curve in $\mathrm{R}$, a language and environment for statistical computing and graphics through the plot function, as shown in Fig. 4. It can be seen from the overall curve, in May 2017, the game was launched globally on App Store and Google Play with large-scale marketing promotion, so the revenue growth is undeniable. Since then, the game has been continuously updated, and game revenue has fluctuated, but the overall performance has stabilized.

\subsection{Preprocess data}

The third action when using our method for game revenue forecast is to pre-process data and check the stationary. For non-stationary time-series, it is necessary to use the method of difference to obtain a stationary time-series. In the $\mathrm{R}$ language, we use the diff () function to perform this operation on time-series. Usually, the time-series changes are mainly

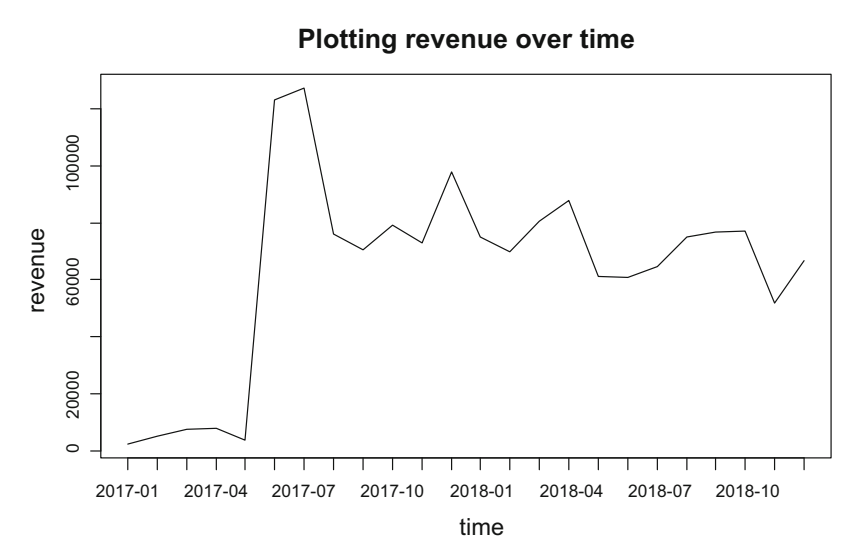

Fig. 4 Game revenue historical performance

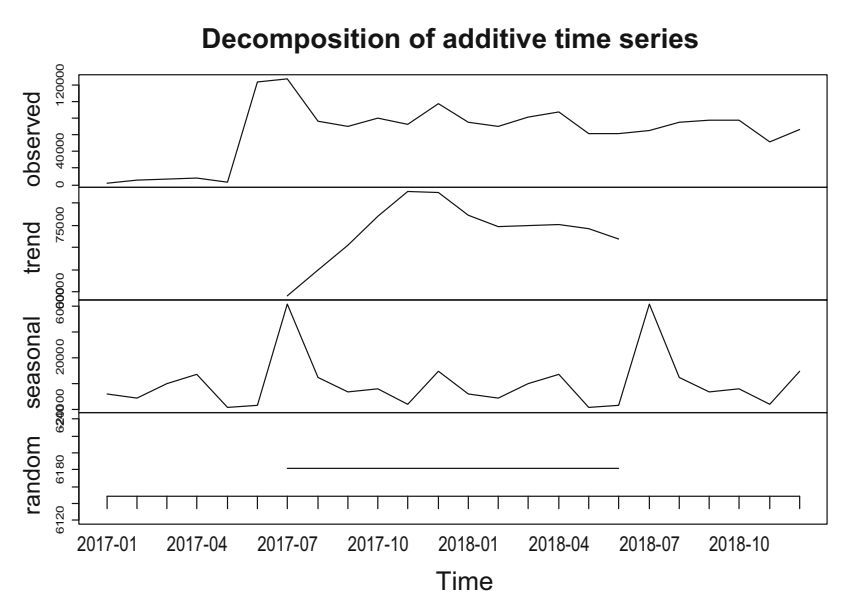

Fig. 5 The decomposition of game revenue

affected by four factors: long-term trend, seasonal variation, cyclical variation, and noise variation. According to the characteristics of the sequence, the revenue can be obtained by adding different variables. As shown in Fig. 5, we decompose the time-series revenue into four parts in the $\mathrm{R}$ language as follows:

\section{Revenue.ts.decompose $<-$ decompose $($ revenue.ts $)$}

It includes the observation of the original time-series, the decomposition trend, seasonal variation, and the noise. As seen from the decomposition, the game revenues have increased significantly since May 2017, mainly due to the game's global launch. From the revenue season change, we can see the revenue increased significantly around July.

\subsection{Apply the prediction}

The fourth action about using our method for the game revenue forecast is to apply the prediction models. Based on the indie game studio's previous player survey, we found that the main players were students who had more time to play games 


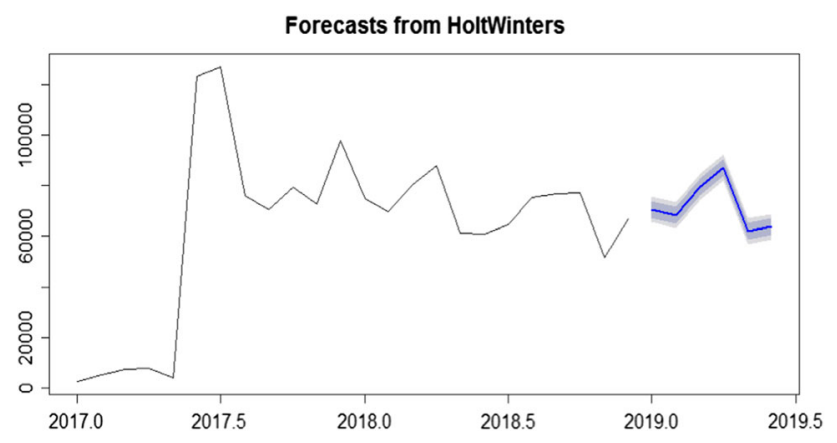

Fig. 6 Revenue forecast by Holt-Winters model

during the holidays. This is a potential reason why revenue has the seasonal trend. So, we use the time-series prediction models for the revenue forecast.

Time-series analysis is a statistical method of dynamic data processing. The typical assumption is that adjacent observations have specific dependencies [30]. The statistical laws of random data sequence compliance are studied based on stochastic process theory and mathematical statistics methods. A stationary time-series is one whose attributes do not depend on how the series is observed [31].

Holt and Winters extended Holt's method to capture seasonality [32]. The Holt-Winters seasonal model comprises the forecast equation and three smoothing equations, including the level $l_{t}$, the trend $b_{t}$ and also the seasonal component $s_{t}$ corresponding smoothing parameters $\alpha, \beta$, and $\gamma$. Through the previous decomposition of the game revenue, especially the seasonal variation, we can see that the game revenue data have obvious seasonality trends. Therefore, the HoltWinters seasonal model can be used for prediction. In the $\mathrm{R}$ language, we can implement revenue forecast by calling the Holt-WintersModel [33], as follows:

Holt - WintersForecast

$$
<- \text { forecast (HoltWinters Model, } h=6, \text { level }=c(80,95))
$$

$h$ represents the number of predicted months. Since the revenue data we have collected is limited, it can only predict the reasonable revenue for six months for this case study. So based on different parameter settings, we finally set the $h$ equal to six. Besides this, we also choose the standard $80 \%$ and $95 \%$ confidence intervals. The confidence interval shows the degree to which the parameter's actual value has a certain probability of falling around the measurement result. As shown in Fig. 6, based on this Holt-Winters seasonal model, we get the prediction about the six-month revenues for the following year.

In order to verify the reliability of our prediction, the autocorrelation function (ACF) and partial autocorrelation function $(\mathrm{PACF}$ ) are analyzed by using the $\mathrm{ACF}$ and the $\mathrm{PACF}$ functions in the $\mathrm{R}$ language.

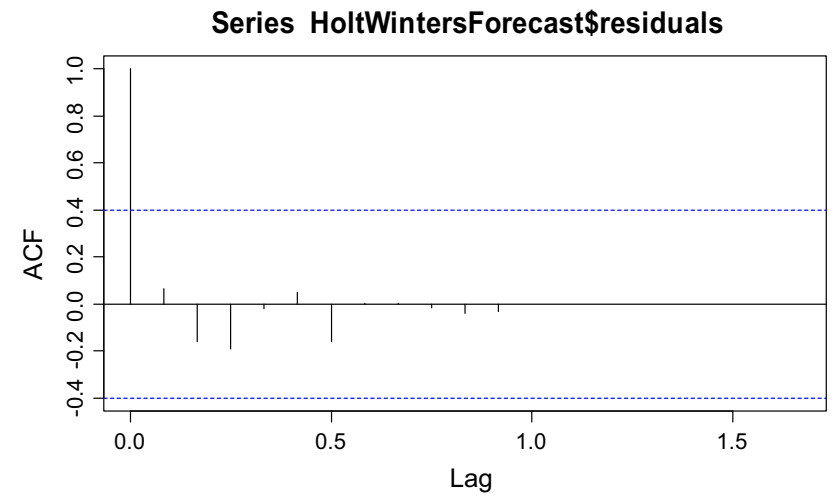

Fig. 7 Holt-Winters ACF plot of residuals

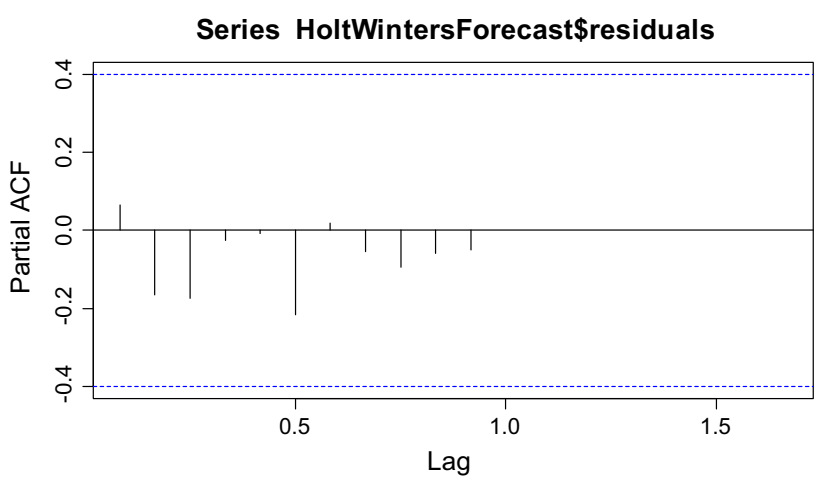

Fig. 8 Holt-Winters PACF plot of residuals

The ACF measures the linear dependence between observations in a time series separated by a lag. The PACF is used to determine how many autoregressive terms are necessary. We can see that there is no significantly non-zero autocorrelation in any lag, so the data are independent and demonstrate a good fit for the model, as shown in Figs. 7 and 8.

In addition, the ARIMA model provides another solution to time-series prediction. Holt-Winters and ARIMA models are the two most widely used approaches to make the time-series prediction. With these two models, we can provide complementary approaches to the revenue forecast. The Holt-Winters model is based on a description of the trend and seasonality in the data. However, the ARIMA model aims to describe the autocorrelations in the data.

The ARIMA was firstly introduced by Box and Jenkins [34]. Since then, this model has been applied in various fields and used in multiple forecast systems [35]. ARIMA characterizes time-series, including three parts. First, the autoregressive (AR) terms model the past process information. Second, the integrated terms (I) that model the differences needed to make the process stationary. Third, the moving average (MA) terms control the past noise information around the process [36].

In practice, we use the $\mathrm{R}$ language to make the prediction by using the ARIMA model. First, we install the forecast 
Forecasts from ARIMA $(1,0,0)$ with non-zero mean

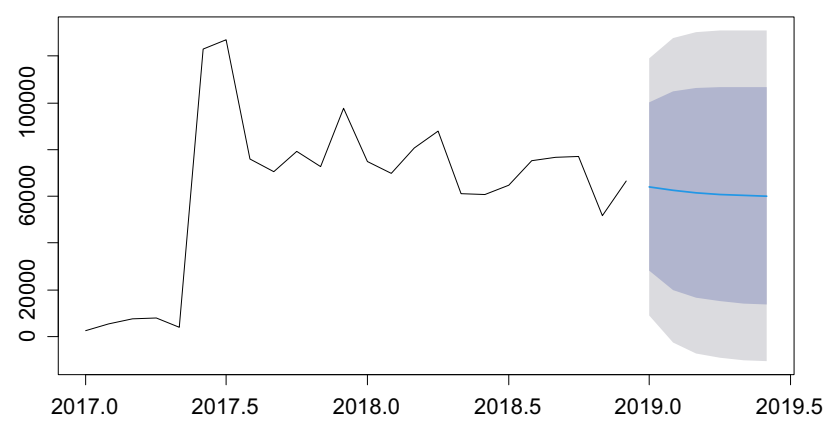

Fig. 9 Revenue forecast by ARIMA model

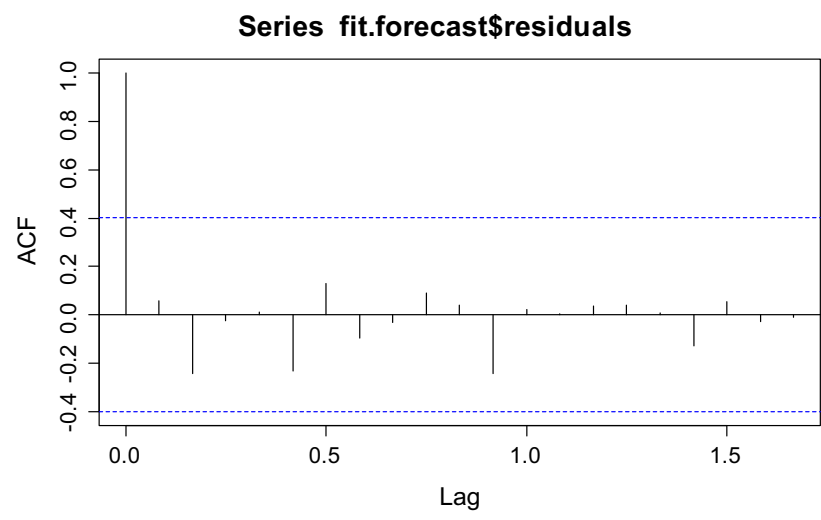

Fig. 10 AIRMA ACF plot of residuals

package with the auto.arima function by default. Second, we use the function to find the suitable $\mathrm{p}, \mathrm{d}, \mathrm{q}$ automatically as follows:

\section{ArimaForecast $<-$ auto.arima $($ revenue.ts $)$}

Third, we predict the next six months' revenue through the forecast function. At last, we use the plot function to draw the prediction graph, as shown in Fig. 9.

Besides this, the ACF and PACF are analyzed by using the $\mathrm{ACF}$ function and the PACF function in the R language. We can see that there is no significantly non-zero autocorrelation in any lag, so the data are independent and demonstrate a good fit for the model, as shown in Figs. 10 and 11.

Hyndman and Koehler [37] point out that errors become the standard measure for forecast accuracy, where the forecast error is scaled by the in-sample mean absolute error obtained using the forecasting method. In practice, three general errors, the Mean Absolute Percentage Error (MAPE), Mean Absolute Error (MAE), and Mean Absolute Scaled Error (MASE), are used to measure the forecast accuracy. In order to compare the forecast accuracy of the two prediction models, we calculated the MAPE, MAE, and MASE both for the ARIMA model and the Holt-Winter model, as shown in Table 1.

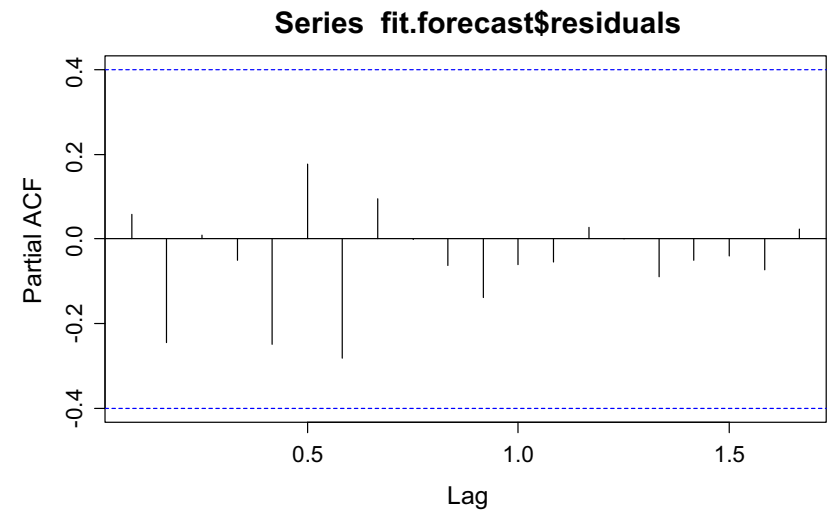

Fig. 11 AIRMA PACF plot of residuals

Table 1 Compared with different prediction models

\begin{tabular}{lll}
\hline Error measure & Holt-winters model & ARIMA model \\
\hline MAPE & 22.6004 & 149.5999 \\
MAE & $15,306.08$ & $18,123.39$ \\
MASE & 0.3438609 & 0.4071537 \\
\hline
\end{tabular}

The error measure comparison of two prediction models shows that the Holt-Winters model is more suitable for predicting the game revenue data, especially with seasonality, so we finally only choose the Holt-Winters model prediction for the reliable benchmark-setting result.

\subsection{Set the benchmark}

The fifth action for our method is to set the revenue forecast as an internal benchmark for the game publishing performance evaluation. Compared with the traditional method based on personal experience [19] and the ARIMA model prediction, we finally set the Holt-Winters forecast revenue as an internal benchmark to drive their mobile game publishing. The main reasons for the revenue forecast are to evaluate if real revenue meets the forecast benchmark and tell the indie game developers what the potential issues of their game publishing are and how to take actions to improve by using our method.

\subsection{Result and contrast}

The sixth action for our method is to track the game publishing real revenue and compare it with our internal revenue benchmark. We compared the benchmark with the real revenue from the indie game studio. As shown in Fig. 12, we plot the real revenue and the internal revenue benchmark in one figure.

According to the forecast revenue trends, we can see that the benchmark made by the Holt-Winters prediction is close to the real revenue. However, for the red ellipse, the real rev- 


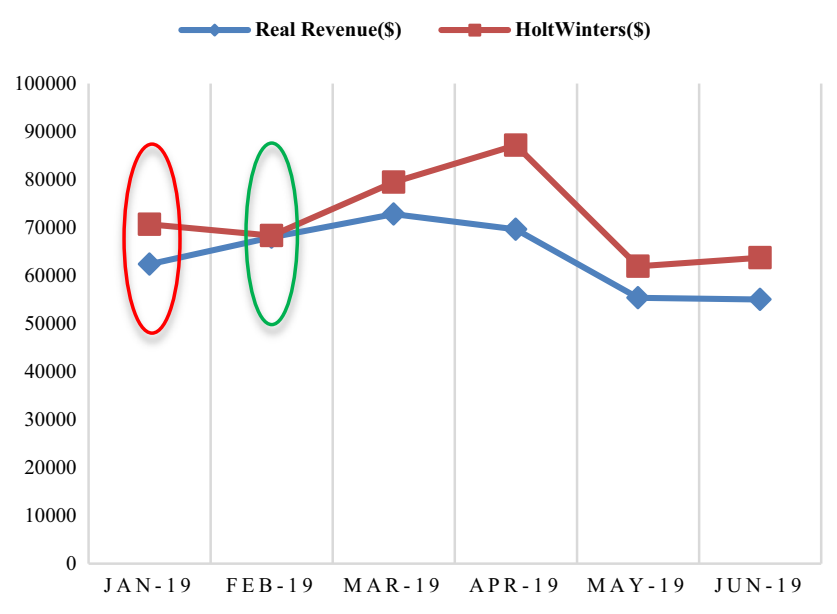

Fig. 12 Holt-Winters forecast and real revenue comparison

enue for January is lower than the benchmark, so we need to analyze the reasons and take actions to drive the mobile game publishing. When the revenue part fails to meet the benchmark, we have to identify what can affect the game revenue during the game publishing. In practice, we need to analyze further and locate potential problems and provide optimization suggestions for indie game developers to improve their game publishing. The real revenue for February (the green ellipse) almost meets our benchmark. That means the game publishing performance is improved to hit the target.

\section{Analysis and suggestion}

According to our case study, the indie game developers did have no experience with game revenue forecast. They never thought that revenue forecast could be used to drive their game publishing process. We guide the indie game developers to set the revenue forecast as an internal benchmark to evaluate game publishing performance. We hold meetings and calculate and discuss whether the monthly revenue has reached our revenue forecast. If it is achieved, it indicates that this month's game version has a good business performance. If it does not meet the expectations, we need to determine the reasons behind the data and take actions to improve by using our method. For example, in January, the revenue was below the benchmark. We get the conclusion that although they released a new version during this month, the revenue performance still cannot meet the benchmark. We need to find out the reason behind the data.

If we dig into the $\mathrm{F} 2 \mathrm{P}$ mobile game publishing process, the new players are mainly recruited through channels like App Store and Google Play. When new players enter the game, they will become active players. Active players can generate game revenue by payment in the game and convert to the paying players. However, as time changes, active players can become inactive players or churn players. Paying players also can change to churn players and inactive players. Based on these player behavior changes during the mobile game publishing, we suggest the indie game studio that needs to consider maintaining active players during the game publishing process and encouraging more active players to pay in the game.

In practice, if the revenue cannot hit the target, it indicates that the indie game developers need further to optimize their game publishing through the new version update, releasing new content to encourage players to pay, and also the marketing promotion that brings more new players and encourages the new players to pay. In addition, the reasons why game revenues can hit the benchmark target also lie in the number of paying players. How to increase the paying player is the key. It depends on the conversion rate from active player to paying player. The ability to make the new content attract and increase the paying players will affect the real revenue. From the new version update side, we suggest that the indie game developer consider developing new content to encourage more players to pay in the game when real revenue cannot reach the benchmark. We gave the indie game developers suggestions such as encouraging non-paying players to pay by first payment rewards to the new players and also recommending the hardcore players with their favorite items to increase their payment. We also suggest the indie game developers avoid the paying players churn by providing more rewards based on their payment.

By using our data-driven method, the indie game developer got the reliable revenue forecast and set the revenue forecast as the internal benchmark to drive their mobile game publishing by comparing it with the benchmark to evaluate their mobile game publishing performance. We also help them find out why the revenue cannot reach the benchmark in January. The reason is that the new version update in January with less attractive content and in-game events, which led few players to pay. So, how to release engaging content and encourage players to pay needs to be solved. They finally accepted our suggestions, made changes in their new game version development. In practice, they add more in-game events and the first payment rewards for the new players, recommend hardcore players with their favorite items and avoid the paying player churn by providing rewards based on their payment and improve the potential issues. Besides this, the indie game studio also gave us positive feedback about our method. After using our data-driven method, they realized it was an effective way to predict the revenue and set the revenue benchmark for each month to benefit them to drive mobile game publishing and save costs. They can quickly get a reasonable expectation about future revenue, which also benefits them to control the marketing budget for different month promotions and track the ROI. 


\section{Conclusion and future work}

The ARM funnel model, which is initially designed for the social game, only includes the acquisition, retention, and monetization stages [18]. It is hard for indie game developers to find out the basic logic of player behavior changes during the mobile game publishing process, such as the changes from new players to active players and paying players. It does not show what player behaviors are related to the revenue and how to increase the revenue by guiding player behavior changes. In this paper, we extend the ARM funnel model for mobile game publishing and focus on solving the revenue problems from the indie game developer side. As for the related prediction research, Runge et al. [20] focus on predicting when a player will leave a game, creating a unique opportunity to increase the player's lifetime and revenue. Periáñez et al. [21] developed a survival integration model and provided comprehensive churn prediction. Sifa et al. [22] focus on predicting players' future purchase behaviors. Xie et al. [23] focus on predicting the first purchase behaviors in two social games. However, the previous game predictions primarily focused on predicting player churn and purchase behaviors. It lacks a direct prediction about game revenue, especially based on the historical revenue data. So we provide the new method to make the revenue forecast only based on the historical revenue and then set the revenue forecast as the internal benchmark to drive mobile game publishing and mainly target the indie game developers.

Revenue forecast and analysis are essential throughout the game publishing process to keep tracking of the ROI, evaluate game version performance, and control the marketing budget. The benefit is to get a reliable prediction based on historical revenue as an internal benchmark and use it to guide actions. Based on our previous interviews with indie game studios, most of them have issues with related game data collection, analysis, and prediction during game publishing [12]. There is a need for a method to support this process based on data collection, analysis, and prediction. We first provided the data-driven method to make revenue prediction based on the time-series prediction models and set it as an internal benchmark to track the mobile game publishing performance. Second, we cooperated with an indie game studio based in Sweden, got involved in one of their projects, and made a revenue forecast for their launched mobile game. Third, we set a reliable forecast revenue as the internal benchmark and compared it with the real revenue. Finally, we provided related analysis and actions to guide the indie game studio to improve their mobile game publishing process. This research can help indie game developers to get internal revenue benchmarks by making a reliable prediction about their game revenue. It uses historical revenue data as input, and the result is then analyzed, and new insights concerning the different publishing activities can be gained.
According to the literature review, the traditional game revenue forecast relies on personal experience [19] that may not be available to a small and medium-sized studio and is unreliable to set as the benchmark. We first provide a method of predicting future revenue based on historical data. Second, we introduce two different time series prediction models into the game field and make the revenue forecast and comparison. Third, by comparing the performance of two different prediction models, we found that the Holt-Winters model is more suitable for predicting data with seasonal changes. As game revenues almost with seasonality, it is more suitable to adopt this model. Finally, we ask the indie game developer to set the revenue forecast as the benchmark to drive the mobile game publishing. In addition, the reasons why game revenues can hit the benchmark target also lie in the paying players. How to increase the paying player is the key. It depends on the conversion rate from active player to paying player. Our research also proposes actions based on data analysis to drive indie mobile game publishing, such as releasing the new content to incentivize active players to pay, recommending hardcore players with their favorite items, and providing rewards to prevent the paying player churn based on their payment and deliver more revenue.

However, our research focuses on helping indie game developers predict mobile game revenue, set it as an internal benchmark, identify existing problems, and provide indie game developers with actions. These actions include optimizing the marketing promotion from the channel side, releasing engaging content to incentivize active players to pay, and providing rewards to prevent the paying player from churning and delivering more revenue. Our method allows the indie game developers to make the revenue forecast only by the historical revenue and then set the revenue forecast as the internal benchmark to drive mobile game publishing. In the future, we will further apply this data-driven method to different indie game studios. With a view to help more indie mobile game developers do revenue forecast themselves, a related revenue forecast tool can be developed for the next step which the indie game developers can upload the historical revenue data and get the forecast directly. Besides this, at present, our evaluation mainly targets the F2P games. It means the mobile games we evaluated all choose free download with in-game payment. However, for P2P (Pay-to-play) games, as its revenue only comes from downloads, there is no meaning to track the player behavior changes related to revenue inside the $\mathrm{P} 2 \mathrm{P}$ game, so further research on driving this kind of game publishing is still needed.

Acknowledgements This research was supported by the University of Skövde, Sweden Game Arena and the Game Hub Scandinavia 2.0 (NYPS 20201849) project under the EU regional development fund Interreg Öresund-Kattegat-Skagerrak.

Funding Open access funding provided by University of Skövde. 
Open Access This article is licensed under a Creative Commons Attribution 4.0 International License, which permits use, sharing, adaptation, distribution and reproduction in any medium or format, as long as you give appropriate credit to the original author(s) and the source, provide a link to the Creative Commons licence, and indicate if changes were made. The images or other third party material in this article are included in the article's Creative Commons licence, unless indicated otherwise in a credit line to the material. If material is not included in the article's Creative Commons licence and your intended use is not permitted by statutory regulation or exceeds the permitted use, you will need to obtain permission directly from the copyright holder. To view a copy of this licence, visit http://creativecomm ons.org/licenses/by/4.0/.

\section{References}

1. Thibault C (2013) Game Data Analysis-Tools and Methods. Packt Publishing Ltd, United Kingdom

2. Newzoo Report, Global Games Market (2020) https://newzoo. com/insights/articles/game-engagement-during-covid-pandemicadds-15-billion-to-global-games-market\%20revenue-forecast/. Accessed 25 Feb 2021

3. Guevara-Villalobos V (2011) Cultures of independent game production: examining the relationship between community and labor. In: proceedings of the 2011 digital games research association conference, think design play. Hilversum, Netherlands

4. Peitz and Waldfogel (2012) The Oxford Handbook of the Digital Economy. Oxford University Press Published, Oxford

5. Davenport TH, Harris JG, Morison R (2010) Analytics at Work: Smarter Decisions, Better Results, 1st edn. Harvard Business Review Press, United states

6. Kim J H, Gunn D V, Schuh E, Phillips B, Pagulayan RJ, Wixon D (2008) Tracking real-time user experience (TRUE): A comprehensive instrumentation solution for complex systems. In: SIGCHI conference on human factors in computing systems. Florence, Italy, pp 443-451

7. Hullett K, Nagappan N, Schuh E, Hopson J (2011) Data analytics for game development (NIER Track). In: 33rd International Conference on Software Engineering. Waikiki, Honolulu, USA, pp 940-943

8. Moura D, El-Nasr M S, Shaw C D (2011) Visualizing and understanding players' behavior in video games: discovering patterns and supporting aggregation and comparison. In: ACM SIGGRAPH symposium on video games. Vancouver. New York, pp 1-6

9. Zoeller G (2013) Game Development Telemetry in Production. In: M. S. El-Nasr, A. Drachen, and A. Canossa, eds. 2013. Game Analytics - Maximizing the Value of Player Data. USA: Springer. Ch. 2

10. Drachen A, Nicholas R, Julian R, Rafet S (2016) Stylized facts for mobile game analytics. In: Conference: IEEE Computational Intelligence in Games (CIG). Santorini, Greece, pp 20-23

11. Michael G (2017) Mobile Revenue Forecasting: A Guide to Not Losing Your Shirt, https://www.gdcvault.com/ play/1024470/ Mobile Revenue Forecasting-A-Guide/. Accessed 15 Jan 2021

12. Su Y, Backlund P, Engström H (2020) Business intelligence challenges for independent game publishing. International Journal of Computer Games Technology

13. Michelle K (2020) what is Data driven? https://www.dataversity. net//what-is-data-driven/. Accessed 17 Jan 2021

14. Freytag PV, Hollensen S (2001) The process of benchmarking, benchlearning and benchaction. TQM Mag 13(1):25-34

15. Elmuti D, Kathawala Y (1997) An overview of benchmarking process: a tool for continuous improvement and competitive advantage. Benchmarking for Quality Management \& Technology
16. Drachen A, El-Nasr M S, Canossa A (2013) Game Analytics - The Basics. In: M. S. El-Nasr, A. Drachen, and A. Canossa, eds. 2013. Game Analytics-Maximizing the Value of Player Data. USA: Springer. Ch. 1

17. Moreira ÁVM, Filho VV, Ramalho GL (2014) Understanding mobile game success: a study of features related to acquisition, retention and monetization. SBC J Interact syst 5:2-13

18. Aaron H (2011) Top 7 Social Game Metrics of 2011. https://www. slideshare.net/kontagent/top-7-social-metrics-gdc-europe-2011/. Accessed 15 Feb 2021

19. Revenue Forecasting for Social and Mobile Gaming Companies, (2012).http://www.financialmodelling.net/tutorials/fiance/revenue Forecasting-for-social-and-mobile-gaming-companies. Accessed 18 Feb 2021

20. Runge J, Gao P, Garcin F, Faltings B (2014) Churn prediction for high-value players in casual social games. In 2014 IEEE conference on Computational Intelligence and Games, pp 1-8

21. Periáñez Á, Saas A, Guitart A, Magne C (2016) Churn prediction in mobile social games: towards a complete assessment using survival ensembles. In: 2016 IEEE international conference on data science and advanced analytics (DSAA), pp 564-573

22. Sifa R, Hadiji F, Runge J, Drachen A, Kersting K, Bauckhage C (2015) Predicting Purchase Decisions in Mobile Free-to-Play Games. In: Proc. of AAAI AIIDE, pp 79-85

23. Xie H, Devlin S, Kudenko D, Cowling P (2015) Predicting Player Disengagement and First Purchase with Event-frequency Based Data Representation. In: Proc. of CIG, pp 230-237

24. Weigend AS (2018) Time series prediction: forecasting the future and understanding the past. Routledge

25. Markovska M, Buchkovska A, Taskovski D (2016) Comparative study of ARIMA and Holt-Winters statistical models for prediction of energy consumption. In: XIII international conference ETAII6, pp $1-6$

26. Montgomery DC, Jennings CL, Kulahci M (2015) Introduction to time series analysis and forecasting. Wiley

27. Van Landeghem R. Persoons K (2001) Benchmarking of logistical operations based on a causal model. Int J Operat Product Manag. pp 254-267

28. Cousin G (2005) Case study research. J Geogr High Educ 29(3):421-427

29. Gerring J (2006) Case Study Research: Principles and Practices. Cambridge University Press, Cambridge, New York

30. Brockwell PJ, Brockwell PJ, Davis RA, Davis RA (2016) Introduction to time series and forecasting. Springer

31. Wei W W (2006) Time series analysis. In: The Oxford Handbook of Quantitative Methods in Psychology: Vol. 2

32. Chatfield C (1978) The Holt-winters forecasting procedure. J Roy Stat Soc: Ser C (Appl Stat) 27(3):264-279

33. RDocumentation (2019) HoltWinters: Holt-Winters Filtering. https://www.rdocumentation.org/packages/stats/versions/3.6.2/ topics/HoltWinters/. Accessed 21 Feb 2021

34. Box GE, Jenkins GM, Reinsel GC, Ljung GM (2015) Time series analysis: forecasting and control. Wiley, United States

35. Lawrence KD, Geurts MD (2006) Advances in business and management forecasting. Emerald Group Publishing, Brigley

36. Fathi O (2019) Time series forecasting using a hybrid ARIMA and LSTM model. Velvet Consulting, pp 1-7

37. Hyndman RJ, Koehler AB (2006) Another look at measures of forecast accuracy. Int J Forecast 22(4):679-688

Publisher's Note Springer Nature remains neutral with regard to jurisdictional claims in published maps and institutional affiliations. 